\title{
Die neue EU-Mitteilung: Rückenwind für die gewerkschaftliche CSR-Politik?
}

\author{
RAINALD THANNISCH
}

Corporate Social Responsibility, kurz CSR, ist ein „Konzept gesellschaftlicher Verantwortung von Unternehmen, das Aspekte der Nachhaltigkeit aufnimmt“.•Dieses Konzept ist in den vergangenen Jahren immer beliebter geworden: bei Unternehmen, Beratungsunternehmen, Wissenschaftlern, Studierenden und nicht zuletzt auch bei der Bundesregierung, die eigens das Nationale CSR-Forum eingesetzt hat. Kein größeres Unternehmen kann sich dem Thema noch entziehen. ${ }^{2}$

Ein Kennzeichen der CSR-Debatte insgesamt ist, dass sie in besonders ausgeprägter Weise mit Hoffnungen und Erwartungen, aber auch mit Befürchtungen verknüpft ist. Aus Sicht der Befürworter wird insbesondere ein doppelter Nutzen von CSR sowohl für die Unternehmen als auch für die Gesellschaft als Ganzes beschrieben: „Die bewusste Auseinandersetzung mit den an das Unternehmen und seine Geschäftstätigkeit gerichteten gesellschaftlichen Erwartungen und Bedürfnissen ist gerade in den Auslandsmärkten von besonderer Wichtigkeit. Strategische Investitionen in die soziale und ökologische Umfeldentwicklung des Unternehmens sind oftmals notwendig, wenn ein Unternehmen erfolgreich wirtschaften und gesellschaftliche Akzeptanz bei Mitarbeitern und Stakeholdern erfahren möchte. “®

Dieser Sichtweise, dem sogenannten Business Case von CSR, wirft der Wirtschaftsethiker Ulrich Thielemann vor, dass sie „ethisch nicht neutral“ब ${ }^{\text {“ }}$ sei, weil sie weiterhin auf das Prinzip der Gewinnmaximierung setze. „Hohe Gewinne erzielen Unternehmen, die entweder gar nichts in Sachen „Ethik“ tun und ebenso solche, die „viel Geld für Umweltund Sozialengagement ausgeben“, die sich dies also leisten können. Und beide Seiten hängen durchaus zusammen, sodass der „Business Case“, der ja ein genereller sein soll, auch diesbezüglich als widerlegt gelten darf. “

Daniel Kindermann wiederum bezeichnet CSR sogar als integralen Bestandteil eines „market liberalism“ ${ }^{\star \bullet}$ im Sinne Margaret Thatchers und Ronald Reagans, gewissermaßen als Ausgleich für einige der sozialen Härten, die im Kontext der Liberalisierung und der Schwächung sozialstaatlicher Institutionen zu verzeichnen seien.

Christoph Sprich hingegen kritisiert die Einbindung sogenannter Stakeholder im Rahmen von CSR-Konzepten als zu weitgehend: „Wenn CSR-Politik darauf hinausläuft, Unternehmen zur Beteiligung von Stakeholdern oder zu bestimmten Verhaltensweisen zu zwingen, weicht das die Eigentumsordnung auf. Räte können und dürfen nicht die Funktion des Privateigentums übernehmen. Dann würde nicht nur der Wirtschaftsstandort, sondern auch die Freiheit Schaden nehmen."

Viele andere Positionsbestimmungen dürften sich im Rahmen des durch die obigen Zitate aufgespannten Bogens wiederfinden. Darunter befindet sich auch die gewerkschaftliche CSR-Politik, die im Folgenden beschrieben werden soll. Im besonderen Fokus steht dabei die Diskussion der jüngst veröffentlichten CSR-Strategie der Europäischen Kommission und die sich daraus ergebenden Handlungsspielräume für Arbeitnehmervertreter und Gewerkschaften.

\section{Gewerkschaftliche Diskussion zu CSR}

Die deutschen Gewerkschaften stehen CSR ,skeptisch, aber nicht ablehnend“ gegenüber. ${ }^{8}$ Die Begrifflichkeit „CSR“
Vgl. Vitols, K. (2011): Nachhaltigkeit - Unternehmensverantwortung - Mitbestimmung, Berlin, S. 7.

(2) Vgl. Birth, S. (2009): CSR - gesellschaftliche Verantwortlichkeit von Unternehmen, in: Hexel, D. (Hrsg.): Never change a winning system, Marburg, S. 171.

(3) Vgl. das von der Bertelsmann Stiftung und dem Auswärtigen Amt initiierte Internetportal: http://www.csr-weltweit. de/service-navigation/ueber-uns/index.nc.html.

(4) Thielemann, U. (2011): Der Business Case - als Gewinnerethik, http://www.mem-wirtschaftsethik.de/blog/blogeinzelseite/article/der-business/.
5 Ebd.

(6) Vgl. Kindermann, D. (2010): Free us so we can do some Corporate Responsibility, in: Ökologisches Wirtschaften (1), S. $19 f$.

(7) Sprich, Ch. (2012): CSR unterwandert Privateigentum und Freiheit, http://www.freiewelt.net/blog-4011/csrunterwandert-privateigentum-und-freiheit.html.

8 Hexel, D. (2011): Deutscher Corporate Governance Kodex ist das Ziel von mehrTransparenz dadurch erreicht und was bewirkt CSR?, in: Ulshöfer, G./Feuchte, B. (Hrsg.): Finanzmarktakteure und Corporate Social Responsibility, Wiesbaden, S. 230 . 
wird nicht offensiv als Slogan oder Motto verwendet. Diese Distanz mag darin begründet sein, dass CSR als Managementkonzept seinen Ursprung in angelsächsischen Ländern hat und dort auch Themenfelder umfasst, die in Deutschland gesetzlich geregelt sind. ${ }^{\odot}$ In der Diskussion wird der soziale Nutzen von CSR dabei insbesondere für die „internationale Dimension von CSR“ gesehen, weil es auf nationaler Ebene bereits ,vergleichsweise starke und verbindliche gesetzliche Schutzbestimmungen, Tarifverträge und Betriebsvereinbarungen zur Sicherung von Arbeitnehmerrechten “(1) gibt.

Bezüglich der Gefahren wird vor allem das sogenannte "green washing" thematisiert, also die Verschleierung sozialer und ökologischer Missstände durch professionell layoutete CSR-Broschüren und ungeprüfte Siegel bzw. Zertifikate. Uwe Wötzel kritisiert darüber hinaus, dass CSR das Primat der Politik infrage stelle: „Diese Politik soll Unternehmen fördern, die freiwillig über die unzureichenden gesetzlichen Regelungen hinaus agieren. Damit soll insbesondere ein Schaufenster für ethisches Investment entwickelt werden. Dieses marktförmige Instrument kann unter Umständen punktuelle, aber nicht strukturelle Verbesse-

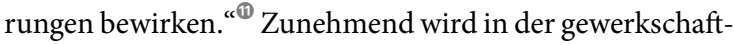
lichen CSR-Debatte aber auch auf die Prekarisierung von Arbeit in Deutschland z. B. durch Leiharbeit oder die missbräuchliche Nutzung von Werkverträgen hingewiesen sowie auf die vermehrten Berichte über die Behinderung von Betriebsratsarbeit bzw. die Verhinderung von Betriebsratsgründungen, offenbar zunehmend auch durch PsychoTricks und Mobbing. ${ }^{\text {[D }}$ Wo solche Verhältnisse herrschen, erübrigt sich eine Diskussion über CSR-Konzepte.

Neben den Risiken von CSR werden in den Gewerkschaften aber auch die Chancen des Konzeptes diskutiert, die darin liegen können, dass sich für engagierte Betriebsräte durch eine Beteiligung an CSR-Strategien Handlungsmöglichkeiten jenseits der institutionellen Grenzen des Betriebsverfassungsgesetzes ergeben. ${ }^{(B)}$ Ein weiterer Ansatzpunkt kann darin bestehen, dass Arbeitnehmervertreter im mitbestimmten Aufsichtsrat CSR thematisieren, beispiels- weise im Rahmen der Innovations- und Personalpolitik oder im Kontext von Risikomanagement und Compliance. ${ }^{(3)}$ Dabei können Aufsichtsratsmitglieder auch darauf hinwirken, dass im Rahmen eines Compliance-Systems die Einhaltung von CSR- oder Ethikrichtlinien überprüft wird. ${ }^{\circledR}$ Von zentraler Bedeutung ist dabei jedoch stets, dass „CSR [...] nicht nur ein werbewirksames Label sein [darf], sondern $[\ldots]$ in eine unternehmerische Gesamtstrategie eingebracht werden [muss]“. ${ }^{\star 1}$

\section{Das 10-Punkte-Papier des DGB von 2009}

Vor dem Hintergrund der auch in den Gewerkschaften differenzierten Diskussion hat der DGB-Bundesvorstand im April 2009 einen Beschluss zum Thema CSR gefasst („10-Punkte Papier“), der im Mai 2010 vom DGB-Bundeskongress bestätigt wurde. ${ }^{\mathbb{D}}$ Der DGB spricht sich darin grundsätzlich für einen Ordnungsrahmen aus, der soziale, ökologische und wirtschaftliche Ziele gleichstellt. Unter anderem fordern die deutschen Gewerkschaften ein „verbindliches internationales Regelwerk zur Durchsetzung sozialer und ökologischer Mindeststandards bei der Globalisierung “ sowie „verbindliche zwischenstaatliche Regelungen der Operationalisierung zur Durchsetzung der [ILO-] Kernarbeitsnormen [...].“

Unternehmerische CSR-Konzepte hingegen können aus gewerkschaftlicher Sicht nur zusätzlich zu gesetzlichen Regelungen einen Nutzen entfalten: „CSR kann über gesetzliche Regelungen hinaus den Schutz der Arbeitnehmerinnen und Arbeitnehmer ergänzen, dies setzt jedoch den Nachweis der Rechtskonformität als Mindestanforderung voraus. Freiwillige CSR-Konzepte können nationale, europäische und internationale Rechtsvorschriften und Tarifvereinbarungen ergänzen, sie aber auch in Zukunft niemals ersetzen. Außerdem darf die Weiterentwicklung und der Ausbau gesetzlicher und tarifvertraglicher Standards nicht behindert werden."

Weiterhin wird aus gewerkschaftlicher Sicht betont, dass die Unternehmen nicht dazu legitimiert sind, ihre Verantwortung gegenüber der Gesellschaft allein zu definie-
(9 Vgl. Vitols, K. (2011): a.a.O., S. 18

(10) Matecki, C. (2007): Vorwort, in: DGB Bundesvorstand/DGB Bildungswerk (Hrsg.): Globalisierung sozial gestalten, Berlin, S. 1.

(1) Wötzel, U. (2011): Geschichte der Unternehmensverantwortung - Primat des Kapitals über die Politik, in: Burckhardt, G. (Hrsg.): Mythos CSR, Bonn, S. 26.

(12) Vgl. für anschaulich dokumentierte Fälle beispielsweise Esser, C./Schröder, A. (2011): Die Vollstrecker, München, S. 46-66 oder den Beitrag von Detlef Esslinger in der SZ vom 27. Februar 2012, S. 17.
(4) Vgl. Feuchte, B. (2011): Unternehmensmitbestimmung und freiwillige Unternehmensverantwortung, in: Ulshöfer, G./ Feuchte, B. (Hrsg.): a.a.O., S. 90ff.

(15) Vgl. Pütz, L. (2011): Compliance, Arbeitshilfe für Aufsichtsräte der Hans-Böckler-Stiftung (15), S. 6, 20.

(16) Vassiliadis, M. (2012), in: Einblick (1), S. 5.

(1) Vgl. Beschluss des 19. ordentlichen DGB-Bundeskongresses: „Nachhaltige Unternehmensverantwortung (CSR) Verbindliche Regeln, die für alle gelten!, http://www.dgb. de/-/a3l. Die folgenden Zitate sind diesem Dokument entnommen. 
ren. Daher wird u. a. gefordert, Mitbestimmungsträger in die Erarbeitung von CSR-Maßnahmen einzubeziehen. „Demokratisch gewählte Arbeitnehmervertretungen müssen an der Ausarbeitung einer unternehmerischen CSR-Strategie beteiligt werden. Eine Debatte um den Umfang und die Folgen von CSR macht für die deutschen Gewerkschaften deshalb nur Sinn, wenn Mitbestimmung und Partizipation der Arbeitnehmerschaft und Gewerkschaften wesentliche Strukturelemente darstellen. Der DGB wird sich für vereinheitlichte, überprüfbare CSR-Standards einsetzen. Nur eine Standardisierung eröffnet denjenigen Unternehmen Wettbewerbsvorteile, die auch gesellschaftlich verantwortungsbewusst handeln, nur eine Vergleichbarkeit erleichtert Verbraucherinnen und Verbrauchern ihre Kaufentscheidung."

\section{Das gemeinsame Verständnis von CSR in Deutschland}

Auf Basis dieser Beschlusslage haben der DGB und die drei im Nationalen CSR-Forum vertretenen Gewerkschaften (IG Metall, ver.di und IG BCE) gemeinsam mit den anderen dort vertretenen Gruppen, darunter Spitzenverbände der Wirtschaft, Nichtregierungsorganisationen (NGOs) und die Bundesregierung, nach langen Verhandlungen ein gemein sames CSR-Verständnis vorgelegt: ${ }^{\circledR}$ "Corporate Social Responsibility (CSR) bezeichnet die Wahrnehmung gesellschaftlicher Verantwortung durch Unternehmen über gesetzliche Anforderungen hinaus. CSR steht für eine nachhaltige Unternehmensführung im Kerngeschäft, die in der Geschäftsstrategie des Unternehmens verankert ist. CSR ist freiwillig, aber nicht beliebig." Von Bedeutung ist hier insbesondere, dass die gesellschaftliche Verantwortung gemäß dieser Definition im „Kerngeschäft“ des Unternehmens wahrgenommen werden muss. Leider zeigt jedoch die Praxis, dass die Auslegung des Satzes „CSR ist freiwillig, aber nicht beliebig" durchaus unterschiedlich ist: Während die Wirtschaftsseite ausschließlich die Freiwilligkeit betont, wird von der Gewerkschaftsseite darauf verwiesen, dass Unternehmen CSR nicht alleine definieren können.

Immerhin bietet das gemeinsame Verständnis eine ganze Auflistung von Kriterien für die Übernahme unternehmerischer Verantwortung, die auch als Argumentationshilfe für die betriebliche Praxis geeignet ist. So heißt es unter anderem: „Unternehmen nehmen gesellschaftliche Verantwortung wahr, indem sie insbesondere:

\footnotetext{
- Mitarbeiterinnen und Mitarbeiter fair behandeln, fördern und beteiligen,

- mit natürlichen Ressourcen schonend und effizient umgehen,

- darauf achten, in der Wertschöpfungskette - in ihrem Einflussbereich - sozial und ökologisch verantwortungsvoll zu produzieren,

- Menschenrechte und die ILO-Kernarbeitsnormen wahren und einen Beitrag leisten, sie international durchzusetzen $[\ldots]^{\prime}$.
}

\section{Praktische CSR-Ansätze von DGB und Gewerkschaften}

Neben der Mitarbeit im Nationalen CSR-Forum sind weiterhin vielfältige praxisnahe Projekte von DGB und Gewerkschaften zur Erhöhung der sozialen und ökologischen Unternehmensverantwortung zu nennen. So engagieren sich die DGB-Mitgliedsgewerkschaften in verschiedenen Branchen-Initiativen und Labels. IG Metall und ver.di beispielsweise sind Mitglieder im CorA-Netzwerk und in der Kampagne für saubere Kleidung. Ver.di, IG BAU und NGG engagieren sich in der Supermarkt-Initiative. IG BAU und IG Metall sind im Forest Stewardship Council vertreten. Die IG BCE hat eine Stiftung Arbeit und Umwelt gegründet und zusammen mit dem Arbeitgeberverband Chemie den Wittenberg-Prozess gestartet, mit dem verantwortliches Handeln in der sozialen Marktwirtschaft gefördert werden soll. Das DGB-Bildungswerk wiederum organisiert seit einigen Jahren ein CSR-Projekt in Vietnam, dessen Ziel darin besteht, Fach- und Führungskräfte vietnamesischer Gewerkschaften und Unternehmen für das Thema „Soziale Verantwortung von Unternehmen “ zu sensibilisieren. ${ }^{(1)}$

Seitens des DGB-Bundesvorstandes wurde Arbeitnehmervertretern im Aufsichtsrat mitbestimmter Unternehmen empfohlen, Ziele gesellschaftlicher Verantwortung in die Ausgestaltung der Vorstandsvergütung zu übernehmen. ${ }^{20}$ Die Partner Bundeskreis Arbeit und Leben, DGBBildungswerk und DGB-Bundesvorstand wollen darüber hinaus die CSR-Debatte in den kleinen und mittleren Unternehmen des Handwerks implementieren. Das hierzu im Rahmen des ESF-Förderprogramms „Gesellschaftliche Verantwortung im Mittelstand“ beantragte Projekt zielt dabei insbesondere auf den Aufbau und die Stärkung ehrenamtlicher Strukturen im Handwerk.

Anerkannte best practice aus Sicht der Gewerkschaften - solange ein verbindliches und überprüfbares internationales Regelwerk fehlt - ist die Vereinbarung sogenannter Internationaler Rahmenabkommen (IRA) durch globale Gewerkschaftsförderationen und multinationale Unternehmen. Diese unterscheiden sich von den unilateral durch

\footnotetext{
(8) Nationales CSR-Forum (2009): Gemeinsames Verständnis von Corporate Social Responsibility (CSR) in Deutschland, http://www.csr-in-deutschland.de/fileadmin/user_upload/ Downloads/ueber_csr/was_ist_csr/Nationales_CSRForum_-_Gemeinsames_Verstaendnis_von_CSR_.pdf. Die folgenden Zitate entstammen diesem Dokument.

(19) Vgl. http://www.nord-sued-netz.de/projekte/vietnam.

(20) Vgl. Seyboth, M./Thannisch, R. (2010): Empfehlungen für eine angemessene Vorstandsvergütung, in: Hans-BöcklerStiftung (Hrsg.): Arbeitshilfen für Aufsichtsräte (14), Düsseldorf, S. $15 \mathrm{ff}$.

(21) Vgl. IG Metall (2006). Umsetzung und Überwachung einer Internationalen Rahmenvereinbarung, Leitfaden, Frankfurt/M.
} 
die Unternehmen definierten codes of conduct u. a. dadurch, dass sie „ausführlichere Bestimmungen über Arbeitsbeziehungen und einen systematischen Bezug auf die ILO-Kernarbeitsnormen" haben und über Streitschlichtungsverfahren verfügen. ${ }^{\text {2 }}$ Eine aktuelle Datenbank der EU-Kommission listet 215 sogenannte transnationale Betriebsvereinbarungen auf, ${ }^{28}$ von denen freilich nicht alle der engeren Definition eines IRAs entsprechen. Dennoch verdeutlicht diese Zahl eindrucksvoll die Dynamik in diesem Kontext.

\section{Neue Dynamik in der CSR-Debatte}

Diese Dynamik zeigt sich derzeit auch in verschiedenen Initiativen. Hier ist das von John Ruggie als UN-Sonderbeauftragter für Menschenrechte und Wirtschaft erarbeitete Rahmenwerk „Guiding Principles on Business and Human Rights“ zu nennen. Dieses Rahmenwerk wurde am 16. Juni 2011 vom UN-Menschenrechtsrat einstimmig angenommen. Unternehmen werden darin unter anderem zu Menschenrechtsverträglichkeitsprüfungen im Rahmen ihrer Sorgfaltspflichten aufgerufen. ${ }^{2}$

Von großer Bedeutung ist auch die von den Gewerkschaften begleitete jüngste Überarbeitung der OECD-Guidelines, mit dem Ergebnis einer übergreifenden operativen Sorgfaltspflicht für die Lieferkette sowie einer Stärkung der Verantwortung für die Einhaltung der Menschenrechte. Eine besonders herausgehobene Bedeutung schließlich kommt der im Oktober 2011 von der EU-Kommission vorgelegten neuen Mitteilung "Eine neue EU-Strategie (2011 - 14) für die soziale Verantwortung der Unternehmen $(\mathrm{CSR}) “ \mathrm{zu}$, die im Folgenden näher vorgestellt werden soll. ${ }^{\text {으 }}$

\section{Die EU-Mitteilung vom Oktober 2011}

In der Mitteilung hat die EU-Kommission CSR nun als „die Verantwortung von Unternehmen für ihre Auswirkungen auf die Gesellschaft ${ }^{{ }^{(2)}}$ definiert. Diese Definition geht deutlich über die frühere Definition der EU hinaus, die CSR ,als ein Konzept [definierte], das den Unternehmen als Grundlage dient, auf freiwilliger Basis soziale Belange und Umweltbelange in ihre Unternehmenstätigkeit und in die Wechselbeziehungen mit den Stakeholdern zu integrieren ${ }^{\text {“ }}{ }^{\text {D }}$ Die Kommission kündigt außerdem an, gegen irreführendes CSR-Marketing vorgehen zu wollen und schlägt vor, soziale und ökologische Kriterien stärker in das öffentliche Auftragswesen einfließen zu lassen. Sie fordert zudem alle in Europa ansässigen multinationalen Unternehmen auf, sich bis 2014 zu verpflichten, die Dreigliedrige Grundsatzerklärung der ILO über multinationale Unternehmen und Sozialpolitik zu beachten. Alle großen europäischen Unternehmen werden aufgefordert, sich bis 2014 zu verpflichten, mindestens entweder die OECD-Leitsätze für multinationale Unternehmen, den Global Compact der Vereinten Nationen oder die ISO-Norm 26000 zur gesellschaftlichen Verantwortung der Unternehmen zu berücksichtigen.

Bei der Entwicklung von CSR sollten die Unternehmen „selbst federführend ${ }^{\text {(ब) }}$ sein. Staatliche Behörden sollten jedoch eine unterstützende Rolle spielen und dabei eine „intelligente Kombination aus freiwilligen Maßnahmen und nötigenfalls ergänzenden Vorschriften einsetzen, die etwa zur Förderung der Transparenz und zur Schaffung von Marktanreizen für verantwortliches unternehmerisches Handeln beitragen und die Rechenschaftspflicht von Unternehmen sicherstellen sollen." Weiterhin plant die Kommission, noch in diesem Jahr eine Rechtsvorschrift zur Transparenz von sozialen und ökologischen Faktoren in der Unternehmensführung vorzulegen.

Diese Vorschläge gehen den Spitzenverbänden der deutschen Wirtschaft deutlich zu weit. So führen sie in ihrer Stellungnahme aus: „Allerdings konterkariert die EU-Kommission diesen praxisorientierten Ansatz in der Mitteilung durch eine Vielzahl von Ankündigungen, die dem freiwilligen Charakter von CSR widersprechen, die Vielfalt von CSR gefährden und zu neuen bürokratischen Regulierungen führen. ${ }^{\text {(8) }}$

Auch die Bundesregierung hat sich im Hinblick auf die angestrebten Berichtspflichten ablehnend geäußert.
22 Schömann, I./Sobcak, A./Voss, E./Wilke, P. (2008): Der Einfluss von Codes of Conduct und Internationalen Rahmenvereinbarungen auf die soziale Regulierung in Unternehmen, Bericht 106 des Europäischen Gewerkschaftsinstituts, Brüssel.

(23) Vgl. http://ec.europa.eu/social/main.jsp?catld=978\&langld= de\&company $=\&$ hdCountryld $=0 \&$ sectorld $=0 \&$ textType $=$ $0 \&$ topicld=0\&keyword $=\&$ mode $=$ searchSubmit.

(24) Vgl. http://www.business-humanrights.org/media/ documents/ruggie/ruggie-guiding-principles-21-mar-2011. pdf.

(25) Vgl. grundlegend: DGB (2011): Die neuen OECD-Guidelines für multinationale Unternehmen - Eine Bestandsaufnahme aus gewerkschaftlicher Sicht, Standpunkt (3), http://www. dgb.de/-/dOI.
26 Vgl. KOM (2011) 681 endgültig, http://ec.europa.eu/ enterprise/newsroom/cf/_getdocument.cfm?doc_id=7008.

(27) Ebd.

(28) $\operatorname{KOM}(2001) 366$.

29. Siehe Fn 26. Das folgende Zitat entstammt ebenfalls dieser Quelle.

30 Stellungnahme von BDA, BDI, DIHK und ZDH zur CSR-Mitteilung der EU-Kommission, http://www.bda-online.de/ www/arbeitgeber.nsf/res/BA2563B7B30D7766C125796F003 1833A/\$file/Stn-CSR-Mitteilung-KOM.pdf. 
In einer Antwort des Bundesministeriums für Arbeit und Soziales an $\mathrm{Ka}$ rin Roth (MdB-SPD) heißt es: „[...] die Bundesregierung [spricht sich] gegen neue gesetzliche Berichtspflichten zu nicht finanziellen (d.h. sozialen und ökologischen) Informationen im Rahmen von CSR aus. Solche gesetzlichen Berichtspflichten würden eine Abkehr vom Prinzip der Freiwilligkeit bedeuten und wären mit erheblichem Bürokratieaufwand insbesondere für kleine und mittlere Unternehmen in Deutschland, aber auch für alle anderen Unternehmensgruppen verbunden. “(

DGB-Vorstandsmitglied Dietmar Hexel hat die neue EU-Mitteilung hingegen als „Schritt in die richtige Richtung“ bezeichnet, dem weitere folgen müssten. In einer im Februar 2012 veröffentlichten Stellungnahme des DGB wird weiterhin ausgeführt, dass die neue Definition von CSR als positive Neuerung betrachtet wird. Auch die angekündigte Rechtsvorschrift zur Offenlegung sozialer und ökologischer Informationen von Unternehmen wäre - so sie in den kommenden Jahren umgesetzt werden sollte - ein wichtiger Schritt in Richtung Transparenz und Überprüfbarkeit. Unzureichend bleibt aus gewerkschaftlicher Sicht jedoch die Einbindung der Arbeitnehmervertreter in die CSR-Strategie sowie das Fehlen internationaler, einheitlicher, überprüfbarer und verbindlicher Regelungen, die notwendig sind, um die Grundsätze gesellschaftlicher Verantwortung von Unternehmen allgemein zu definieren und durchzusetzen. Insgesamt bleiben die Forderungen der EU-Kommission gegenüber den Unternehmen für den DGB zu vage und zu unverbindlich. ${ }^{3}$ Auch der Europäische Gewerkschaftsbund (EGB) hat die neue EU-Strategie grundsätzlich begrüßt, aber konkrete und verbindlichere Maßnahmen gefordert. ${ }^{\text {(2) }}$

\section{Optionen für die gewerkschaftliche CSR-Politik}

Zusammenfassend wird deutlich, dass Gewerkschaften vielfältig engagiert sind, um die Wahrnehmung sozialer und ökologischer Verantwortung durch die Unternehmen zu erhöhen, dass diese Initiativen jedoch nur teilweise unter der mitunter kritisch diskutierten Begrifflichkeit „CSR“ umgesetzt werden. Deutlich wird aber auch, dass derzeit im Themenfeld CSR eine neue Dynamik zu verzeichnen ist, die nicht unwesentlich durch die neue EU-Mitteilung getragen wird. Diese neue Dynamik geht in Richtung von mehr Transparenz, mehr Überprüfbarkeit und mehr unternehmerischer Verantwortung (gemessen an internationalen Standards). Vom bislang vorherrschenden Dogma der Freiwilligkeit wird vorsichtig abgerückt.

Auch wenn die Inhalte der EU-Mitteilung noch deutlich hinter den gewerkschaftlichen Vorstellungen zurückbleiben, so können sie dennoch eine wichtige Argumentations- und Handlungsgrundlage für Arbeitnehmervertreter und Gewerkschaften bieten, um ihre Ansprüche an die soziale und ökologische Verantwortung von Unternehmen umzusetzen.

Fraglich ist jedoch, ob dieser „Rückenwind“ aus der internationalen und vor allem europäischen Debatte auch zu einer Überarbeitung der CSR-
Politik der Bundesregierung führen wird. So hat der DGB die Bundesregierung aufgefordert, „gemeinsam mit den Mitgliedern des CSR-Forums einen konstruktiven Beitrag zur Umsetzung und Weiterentwicklung der EU-Strategie zu leisten. Ein Beitrag dazu könnte darin liegen, den Aktionsplan CSR der Bundesregierung so weiterzuentwickeln, dass er der von der EU-Kommission geforderten intelligenten Kombination aus freiwilligen Maßnahmen und (nötigenfalls) ergänzenden Vorschriften entspricht und die UN-Leitprinzipien berücksichtigt. “囦

Angesichts der oben beschrieben erheblichen Interessengegensätze zwischen NGOs und Gewerkschaften einerseits und Arbeitgeber- und Wirtschaftsverbänden sowie der Bundesregierung anderseits ist in dieser Frage derzeit jedoch kein Konsens erkennbar.

Immerhin hat das Nationale CSR-Forum einvernehmlich die Einsetzung einer Arbeitsgruppe „CSR im europäischen und internationalen Kontext" beschlossen, die bis zum Sommer 2012 hinsichtlich der Umsetzung der neuen EU-Strategie auf nationaler Ebene eine Empfehlungsvorlage für das CSRForum erarbeiten soll. ${ }^{\bullet}$ Auf die Ergebnisse darf man gespannt sein!

(31) Antwort des BMAS vom 15.02.2012 auf die schriftliche Frage Nr. 85 im Februar 2012.

(32) Hexel, D. (2012): Neue EU-Strategie für soziale Verantwortung von Unternehmen - Schritt in die richtige Richtung, in: Arbeitsrecht im Betrieb 33 (1), S. 1.

33 Vgl. Stellungnahme des DGB zur CSR-Mitteilung der EU-Kommission vom 25.10.2011, demnächst im Internet veröffentlicht unter: www.dgb.de.

(34) EGB-Entschließung über eine neue EU-Strategie(2011-14) für die soziale Verantwortung der Unternehmen (CSR), http://www.etuc.org/a/9432.

(35) Stellungnahme des DGB zur CSR-Mitteilung der EU-Kommission vom 25.10.2011, a.a.O.

36 Vgl. Newsletter zur Umsetzung des Aktionsplans CSR der Bundesregierung 2. Ausgabe März 2012.

\section{AUTOR}

RAINALD THANNISCH, Diplom Volkswirt, arbeitet in der Abteilung Mitbestimmungspolitik beim DGB Bundesvorstand und vertritt den DGB in Arbeitsgruppen des Nationalen CSR-Forums.

rainald.thannisch@dgb.de 